\title{
Interhemispheric Coordination of Premotor Neural Activity during Singing in Adult Zebra Finches
}

\author{
Eric T. Vu, ${ }^{1}$ Marc F. Schmidt, ${ }^{2}$ and Mark E. Mazurek ${ }^{2}$ \\ ${ }^{1}$ Division of Neurobiology, Barrow Neurological Institute, Phoenix, Arizona 85013, and 2Division of Biology, California \\ Institute of Technology, Pasadena, California 91125
}

\begin{abstract}
The song system, a neural network that mediates the learning and production of song by oscine songbirds, is investigated extensively as a model system for understanding the neural basis of complex skill learning. Part of the complexity of birdsong arises from the coordinated recruitment of multiple groups of muscles on both sides of the body. Although the song system is bilaterally organized, little is known about how premotor activities on the two sides are coordinated during singing. We investigated this by unilaterally recording neural activity in the forebrain song nucleus HVc (also known as the high vocal center) during singing and by forcing the premotor activities in the two hemispheres out of synchrony by perturbing neural activity in the contralateral HVc with electrical stimulation. Perturbing the activity in one HVc at any time during a song led to
\end{abstract}

a short-latency readjustment of activity in the contralateral HVc. This readjustment consisted of a true resetting of the temporal pattern of activity in the contralateral HVc rather than merely a transient activity suppression overlaid on an unaltered pattern of premotor activity. These results strongly suggest that the output of song premotor areas in the forebrain is continuously monitored and that an active mechanism exists for resynchronizing the outputs from the two hemispheres whenever their gross temporal patterns differ significantly. The possible anatomical substrates for these coordinating mechanisms and their potential roles in song learning are discussed.

Key words: birdsong; song system; zebra finch; interhemispheric coordination; song learning; motor coordination; motor learning
Birdsong is a complex learned behavior that requires the bilateral coordination of many different muscles involved in controlling the vocal apparatus and respiration (Hartley, 1990; Suthers, 1990; Goller and Suthers, 1995, 1996a). A network of discrete brain nuclei collectively known as the song system (Fig. 1) controls these muscles to produce song. These nuclei are found on both the right and left sides of the brain and are anatomically indistinguishable between sides, suggesting that the song control network is a bilaterally symmetrical system. Although it is generally thought that neural activity on both sides of the song system contributes to song production, very little is known about how the activities on the two sides are coordinated with each other. The present paper begins to address this question by investigating the nature and degree of coordination of the premotor activities in the two hemispheres during singing.

Recent data indicate that neurons in the forebrain song nucleus $\mathrm{HVc}$ (also known as the high vocal center) participate directly in specifying the temporal pattern of song (Vu et al., 1994). Thus, temporally specific motor commands are thought to descend from the forebrain during singing (McCasland, 1987; Yu and Margoliash, 1996), and it is likely that the commands descending from

\footnotetext{
Received April 9, 1998; revised Aug. 11, 1998; accepted Aug. 14, 1998.

This work was supported by an Alfred P. Sloan fellowship to E.T.V. and by the following National Institutes of Health grants: the National Research Service Award (NRSA) NS08915 and R29 MH57065 to E.T.V. and the NRSA DC00125 and R03 DC03041 to M.F.S. We thank P. Drew and P. Sule for technical assistance and Drs. G. Laurent and M. Konishi for helpful comments on this manuscript. We also wish to thank M. Konishi for his generous support and for the use of his laboratory.

Correspondence should be addressed to Dr. Eric Vu, Division of Neurobiology, Barrow Neurological Institute, St. Joseph's Hospital and Medical Center, 350 West Thomas Road, Phoenix, AZ 85013.

Dr. Mazurek's present address: Department of Physiology and Biophysics, Regional Primate Research Center, University of Washington, Seattle, WA 98195.

Drs. E.T. Vu and M.F. Schmidt contributed equally to this paper.

Copyright (C) 1998 Society for Neuroscience $\quad 0270-6474 / 98 / 189088-11 \$ 05.00 / 0$
}

the two hemispheres must be precisely coordinated with each other to give rise to the exquisite bilateral coordination of muscle activity observed in the vocal apparatus during singing (Suthers, 1990; Allan and Suthers, 1994; Goller and Suthers, 1995). However, it is not clear whether any of this coordination occurs at the level of the forebrain because neither the HVc nor any other forebrain song nucleus projects to its contralateral homolog.

In theory, continuous interhemispheric coordination of premotor activity during singing might not be required if song is the product of two independent but highly stereotyped motor programs, one in each hemisphere, that simply need to be synchronously initiated to remain in synchrony. Alternatively, both synchronous initiation and feedback mechanisms might be involved in interhemispheric coordination, with the feedback mechanisms serving to resynchronize the two hemispheres after detection of a sufficiently large mismatch between premotor signals descending from the two hemispheres during singing.

The goal of the present study was to test directly whether feedback mechanisms exist to coordinate the premotor activities in the two hemispheres continuously during song. We have recorded the neural activity in one HVc (on either side) during singing, and we have forced the premotor activities in the two hemispheres out of synchrony by perturbing the neural activity in the contralateral $\mathrm{HVc}$ with electrical stimulation. If the two hemispheres act independently once they are initiated synchronously, then this manipulation should not alter the activity pattern in the recorded (and unstimulated) HVc. In contrast to this prediction, we found that perturbing the activity in one $\mathrm{HVc}$ during singing led to a short-latency readjustment of activity in the contralateral HVc. These results indicate that premotor activities in the two hemispheres are indeed coordinated continu- 


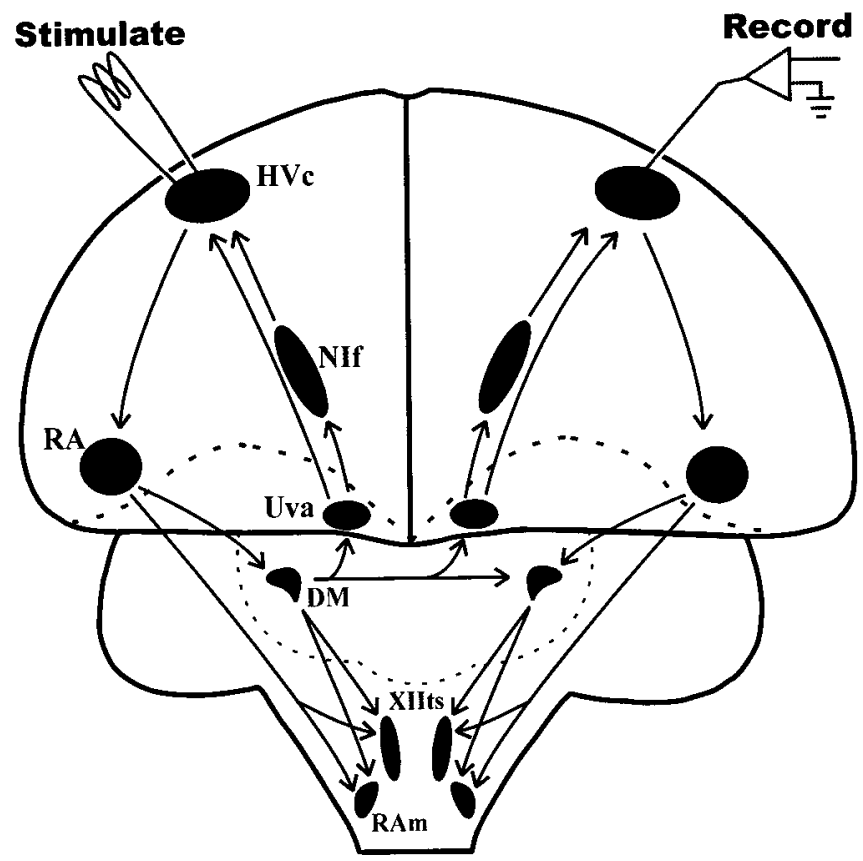

Figure 1. Song production circuit and electrode placement. Schematic diagram of the bilateral neural network for song production (arrows, anterograde projections). Note that no connection across the midline exists between any of the song control nuclei in the forebrain (top two lobes), whereas as many as three bilateral projections exist from a song nucleus below the forebrain to one in the forebrain. (For clarity, only the bilateral $D M$ to $U v a$ projections are shown; the other two known bilateral feedback pathways are described in the Discussion.) Also for the sake of clarity, contralateral projections from the right $D M$ as well as sparse descending projections from $R A$ and $D M$ to contralateral song nuclei in the brainstem are omitted. A pair of stimulating electrodes was implanted chronically in one $H V c$ on either side, and a recording electrode was implanted chronically in the contralateral HVc. DM, Dorsomedial nucleus of the intercollicular region; $H V c$, the acronym is used as the name of this nucleus; NIf, interfacialis of the neostriatum; $R A$, robustus archistriatalis; $R A m$, retroambigualis; Uva, uvaeformis; XIIts, tracheosyringeal part of hypoglossal nucleus.

ously during song and suggest specific mechanisms for such coordination.

Parts of this paper have been published previously (Vu et al., 1996).

\section{MATERIALS AND METHODS}

Animals and surgery. Adult ( $>120 \mathrm{~d}$ after hatching) male zebra finches (Taeniopygia guttata) were obtained from our breeding colony or from a commercial breeder (Magnolia Bird Farms, Anaheim, CA). To increase the frequency of singing behavior, we implanted these birds subcutaneously with small pellets of testosterone proprionate in Silastic (Gurney, 1981) several days before surgery. A total of seven birds were used in these studies; four birds were recorded on the left side and stimulated on the right side, and three birds were stimulated on the left side and recorded on the right side. For chronic implantation of recording and stimulating electrodes, birds were anesthetized with a mixture of ketamine $(25 \mathrm{mg} / \mathrm{kg})$ and xylazine $(5 \mathrm{mg} / \mathrm{kg}$, i.m.) and placed in a stereotaxic device with a custom-built bill bar to hold the upper mandible at $30^{\circ}$ below horizontal. Body temperature was maintained at $38^{\circ} \mathrm{C}$ throughout the surgery. The skull was exposed, and small holes were made over the brain areas to be implanted with electrodes. The junction of the cerebral hemispheres and the cerebellum served as our fiduciary mark. For implantation in the $\mathrm{HVc}$, typical coordinates with respect to this reference point were $0.0 \mathrm{~mm}$ anterior, $2.3 \mathrm{~mm}$ lateral, and $0.3 \mathrm{~mm}$ depth. Each electrode to be implanted was lowered into the nucleus while monitoring neural activity; criteria for determining whether the electrode was correctly placed into the $\mathrm{HVc}$ during surgery included bursty neural activity and evoked auditory responses that exhibited selectivity to the bird's own song (Margoliash and Fortune, 1992; Lewicki, 1996). Two electrodes (separated by $300-400 \mu \mathrm{m}$ ) were placed into each HVc of each experimental bird. All electrodes for each bird were connected to a nanoconnector (Ultimate, Orange, CA), and the whole assembly was cemented (GripCement, Milford, DE) onto the bird's skull with the nanoconnector placed several millimeters away from any implanted electrode. A stainless steel wire was also inserted under the scalp and partly cemented to the skull to ground the animal.

Electrodes. Both stimulating and recording electrodes were fabricated from Formvar-insulated nichrome wires $(25 \mu \mathrm{m}$ bare diameter; AMSystems, Seattle, WA) with tips that were electroplated with gold or rhodium to lower the tip impedance. Typical impedances of electrodes ranged from $100 \mathrm{k} \Omega$ to $1 \mathrm{M} \Omega$ (measured at $1 \mathrm{kHz}$ ). Stimulation electrodes were always implanted in pairs that were 300-400 $\mu \mathrm{m}$ apart. Stimulation currents were applied across such electrode pairs and were isolated from ground.

Experimental sessions. After electrode implantation, birds were kept isolated in sound-attenuating chambers (Industrial Acoustics Company, New York, NY) for several days before being subjected to a single recording session. They were provided with food, gravel, and water ad libitum. At the beginning of the experimental session, the bird was placed in a cage within a sound-attenuated recording chamber and attached to a commutator via a flexible cable made from a bundle of seven Tefloncoated stainless steel wires $(200 \mu \mathrm{m}$ bare diameter). This cable was attached to an ultra-lightweight, low-noise, LinCMOS, operational amplifier (TLC27L4B; Texas Instruments, Dallas, TX) that was connected to the bird's head. This amplifier served as a unity-gain voltage follower that provided a low-impedance path from the bird's head to the main recording amplifier, thus greatly reducing movement artifacts from the signal. The flexible cable and commutator allowed full freedom of movement while providing electrical connections between the bird and the recording and stimulating circuitry. Neural signals and sounds recorded by a microphone placed $30 \mathrm{~cm}$ from the recording cage were digitized and stored with a computer (SPARC Station; Sun Microsystems, Palo Alto, CA) equipped with a data acquisition system (Proport Model 656; Ariel). Both sound and neural records were digitized at a 32 $\mathrm{kHz}$ sampling rate and 16-bit resolution and bandpass filtered between $400 \mathrm{~Hz}$ and $10 \mathrm{kHz}$.

Electrical stimuli to the brain were generated and isolated from ground using an AM-Systems 2100 stimulator. Stimuli were manually triggered during singing and consisted of a train of two to seven biphasic pulses at $400 \mathrm{~Hz}$ with a pulse duration of $0.4 \mathrm{msec} / \mathrm{ph}$ ase. The choice of stimulus current amplitudes to use (40-50 $\mu \mathrm{A})$ was based on a previous study ( $\mathrm{Vu}$ et al., 1994), in which stimuli of similar intensities applied unilaterally to the $\mathrm{HVc}$ were sufficient to perturb the ongoing song pattern, whereas stimuli applied just outside the HVc (by as little as $150 \mu \mathrm{m}$ ) did not.

Only one recording session (lasting 3-5 hr) was used for each bird to minimize variation in the pattern of premotor activity across songs at the same recording site caused by uncontrollable factors such as slight movement of the electrode tip or increasing gliosis near the tip across days. A female zebra finch was placed in an adjacent cage at the start of the session to induce directed singing (Sossinka and Böhner, 1980).

Histology. To locate the sites of implanted electrode tips, we deeply anesthetized the birds with a mixture of ketamine and xylazine (same dosage as for surgery) and perfused transcardially with $0.9 \%$ saline followed by $2 \%(\mathrm{v} / \mathrm{v})$ formaldehyde. The chronic electrodes were postfixed inside the brain in $2 \%$ formaldehyde for $48 \mathrm{hr}$ and then placed in $30 \%$ sucrose in phosphate buffer for 5-7 d for cryoprotection. The electrodes were carefully removed from the brain before the brain was removed from the skull. Parasagittal sections of $40 \mu \mathrm{m}$ thickness were cut on a freezing microtome, mounted, and stained with cresyl violet. The locations of individual recording or stimulating electrodes were identified based on the significant amount of gliosis present around the tip of the electrode. In several cases, it was deemed that electrodes had not been left in the bird for a long enough period for gliosis to occur. In those cases, a small lesioning current was applied 2-3 d before perfusion. Lesion sites were readily identified as small, darkly stained spherical areas.

Data analysis. Digitized data were analyzed off-line using the commercial programs Matlab (The MathWorks, Natick, MA) and Origin (Microcal, Northampton, MA). Using the conventions of Sossinka and Böhner (1980), we characterized a zebra finch song (or "strophe") as a group of vocalizations that is preceded by at least $2 \mathrm{sec}$ of silence and that begins with a set of three or more introductory notes. For quantification 

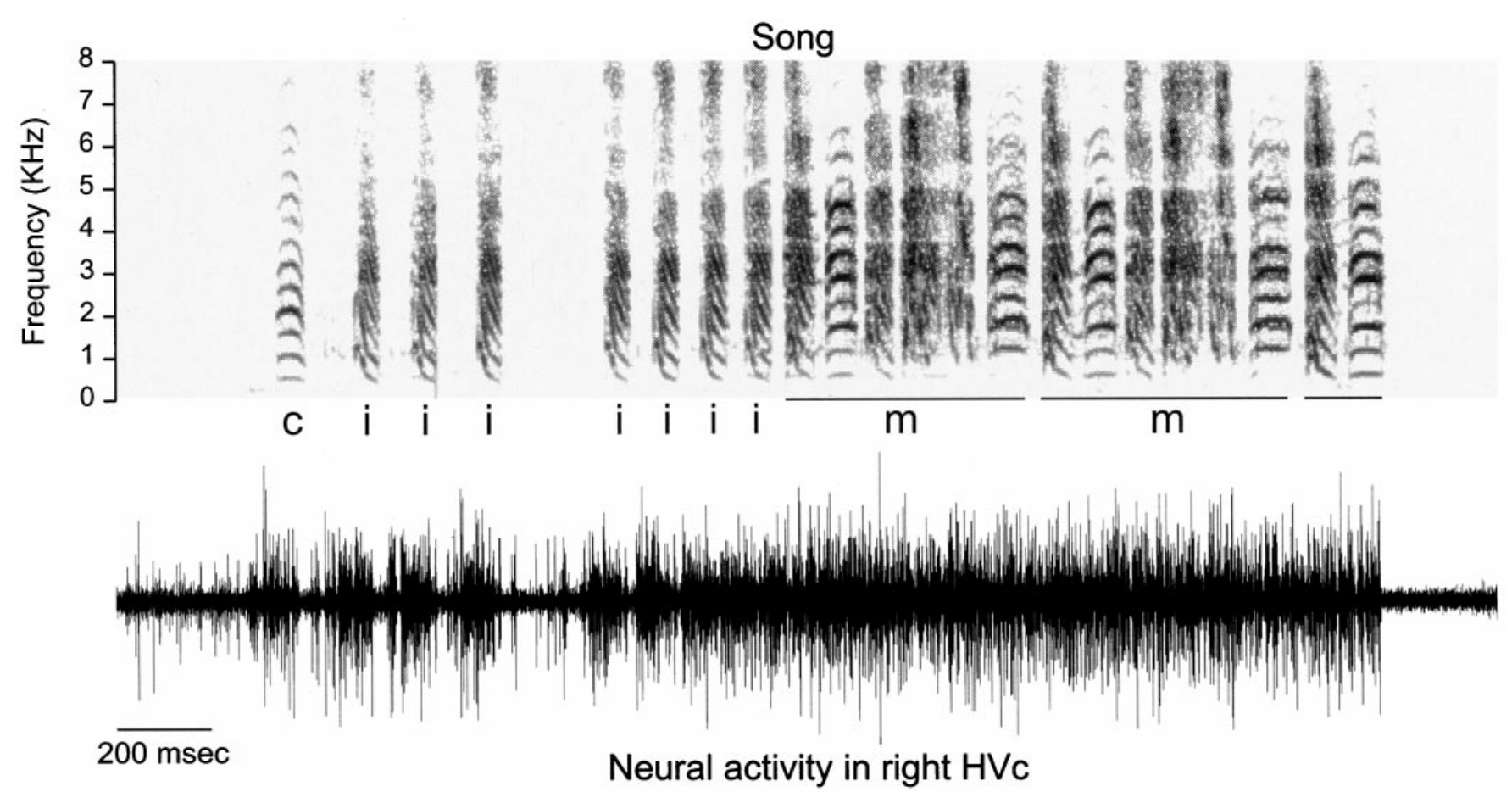

Figure 2. Premotor neural activity recorded in the nucleus HVc during singing. Top, Spectrogram (sound frequency spectrum vs time) of the song of an adult male zebra finch. Bottom, The multiple-unit activity recorded in the right $\mathrm{HVc}$ of the same bird simultaneously. A song typically consists of a series of introductory notes $(i)$ followed by one or more repetitions of a motif $(m)$, a stereotyped sequence of syllables (underlined). A bird may stop singing without having completed the last motif. Vigorous bursts of multiunit activity in the HVc were time-locked to each introductory note [and chirps $(c)$ ] with burst onsets leading note onsets by $50-70 \mathrm{msec}$. Neural activity remained elevated during song motifs and abruptly diminished at song termination.

of the stereotypy of the pattern of neural activity in the HVc during singing, song and neural records were segmented into their component "motifs" or phrases, which are the minimal sequences of syllables that are repeated within the songs. For each bird, 7-14 (control) motifs sung without brain stimulation and their corresponding $\mathrm{HVc}$ premotor activity were analyzed and compared with the premotor activity associated with 2-5 motifs during which a stimulus was applied. We obtained a total of 29 neural records containing a stimulus; in all of which the associated motif was suspended. Singing immediately resumed after a majority of these stimuli (18/29), making it possible to compare the pattern of the resumed neural activity with that from unstimulated motifs. The remaining stimuli either were followed by song termination $(5 / 29)$ or occurred too late in the motif $(6 / 29)$ to allow a comparison between resumed and normal activity.

Neural records were full-wave rectified and subsequently smoothed by convolving the waveform with a window in the shape of a Gaussian density function with a SD of $3 \mathrm{msec}$ (approximate width of $12 \mathrm{msec}$ ). The mean baseline activity level at each recording site was calculated from a nonsinging period (1.75-3.125 sec long) during the same session. All neural activity levels during singing were normalized by subtracting this baseline level. To create peristimulus time histograms, we transformed raw neural records into multiunit rasters. Individual action potentials were defined as peaks in the waveform above a threshold defined as 3 SDs above the mean activity level measured at the same recording site during nonsinging periods.

Statistical analyses used were paired $t$ tests; all mean data are expressed with their corresponding SEMs. Analysis of correlations between waveforms used the Pearson's correlation coefficient.

\section{RESULTS}

\section{Normal song premotor activity in the nucleus HVc exhibits a recurring pattern}

Seven adult male zebra finches were permanently implanted with fine electrode wires in both the left and right $\mathrm{HVc}$, and neural activity from small clusters of neurons was recorded in the freely behaving bird during singing. Neural activity was also recorded in response to presentation of auditory stimuli during nonsinging periods. In all recorded birds, HVc neurons fired vigorously during various vocalizations (calls, chirps, and songs; Fig. 2) but failed to show any response to playback of auditory stimuli, including playback of the bird's own song, during the awake recording session (data not shown). The absence of auditory responsiveness, as well as the fact that vocal output was always preceded by significantly increased neural activity, suggests that we were recording from clusters of premotor neurons.

To determine the effects of electrically stimulating one $\mathrm{HVc}$ on the pattern of neural activity in the contralateral $\mathrm{HVc}$, it was necessary first to characterize the normal pattern of neural activity in the HVc during singing. This allowed us to compare for each recording site the neural activity during normal singing with that observed immediately after stimulation of the contralateral HVc. Figure 2 illustrates the typical pattern of activity observed in the HVc during singing. As has been reported by others (McCasland, 1987; Yu and Margoliash, 1996), we observed a pattern in this activity that was related to the recurrence of syllable types within the song and consequently to the recurrence of the motif (a stereotyped sequence of syllables) [compare with Fig. $1 b$ in $\mathrm{Yu}$ and Margoliash (1996)].

The stereotypy of the neural pattern in relation to the song motif was confirmed by obtaining segments of the neural record corresponding to individual motifs and aligning these segments to the onset of the first syllable of the motif in the associated sound records. In Figure 3, individual neural records were processed by rectification and smoothing of the raw neural records (see Mate- 

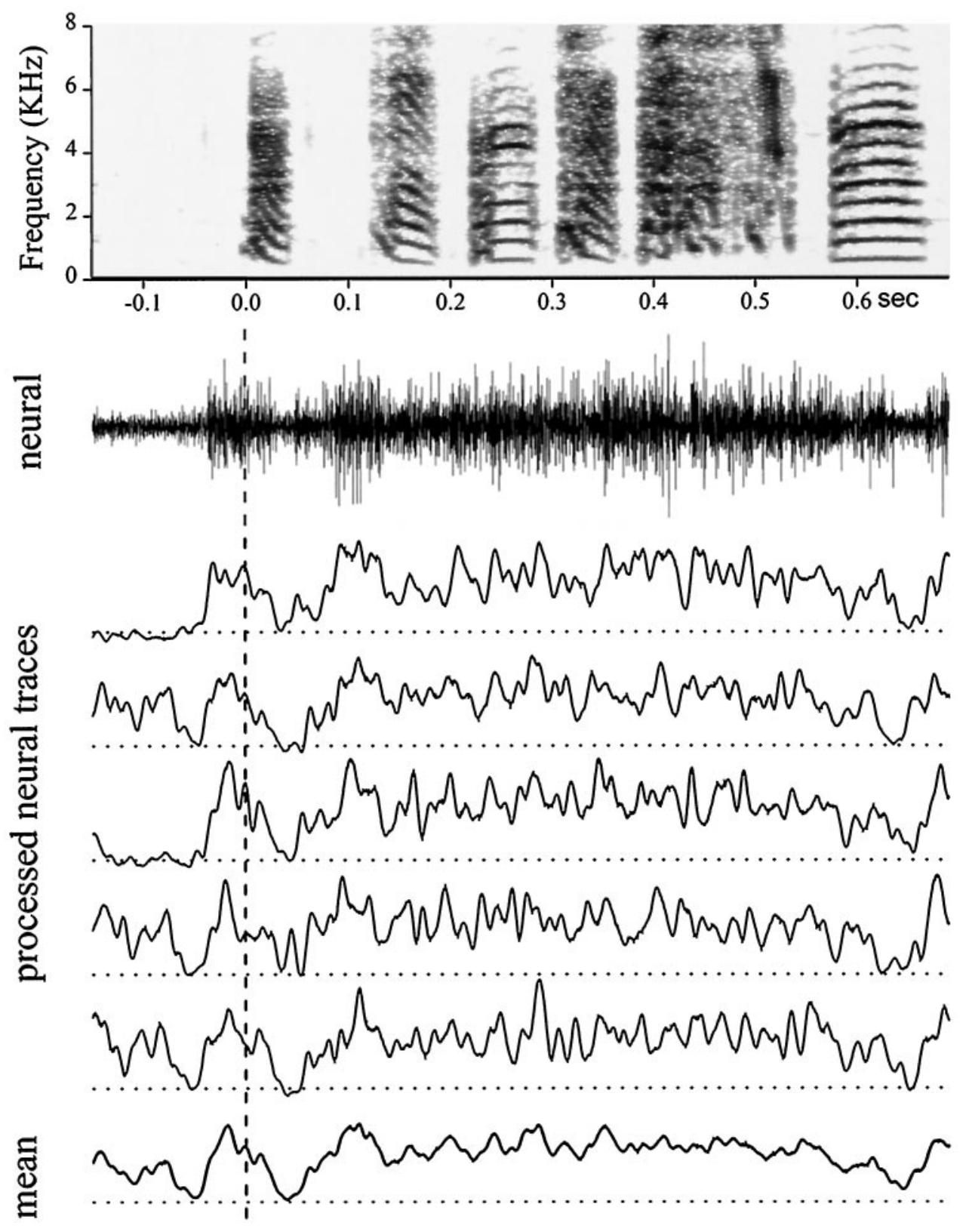

Figure 3. Premotor activity in the $\mathrm{HVc}$ is stereotyped across motifs. Top, The spectrogram of one motif of a zebra finch song is shown. Neural trace, The trace immediately below the spectrogram is the neural activity recorded simultaneously in the right HVc of this bird. Processed neural traces, The next trace represents the result of processing the above neural trace by rectification and smoothing (see Materials and Methods). The horizontal dotted lines in this and other traces indicate the mean background level of activity. Four additional processed neural traces associated with four other motifs sung by the same bird are shown, aligned in time to the beginning of the motif in their associated sound records (vertical dashed line). Bottom, Trace was produced by averaging 10 such processed and aligned neural traces. rials and Methods). For each recording site, a "mean" neural trace was obtained by aligning and averaging 4-10 such processed neural records (Fig. 3, bottom). The resulting mean record was nonmonotonic, contained clear activity peaks, and was consistently above baseline activity level for all nine recording sites in seven birds.

To determine how well the mean neural trace represented a consistent activity pattern across motifs, we compared individual neural traces from three randomly selected unstimulated motifs with the mean neural trace generated at the same recording site. All traces at each recording site were aligned using the first syllable of the motif, and the linear correlation coefficient $r$ was obtained between each individual and the mean neural trace. To reflect the fact that premotor activity was being assessed, comparisons were made from $50 \mathrm{msec}$ before the start of the motif to $30 \mathrm{msec}$ before the mean end of unstimulated motifs. The three correlation values obtained at each recording site were averaged. The mean correlation across all nine recording sites was $0.72 \pm$
0.03 ( \pm SEM). In contrast, if the individual traces were shifted forward in time by $36 \mathrm{msec}$ (three times the size of the window used to smooth the neural records; see Materials and Methods) relative to the mean trace, the resulting mean correlation across all recording sites was $-0.06 \pm 0.04$ (aligned vs shifted; $t_{8}=$ 20.335; $p<0.001$ ). The high degree of correlation between mean and individual traces at each recording site and the marked sensitivity of this correlation to a small time shift indicate that a consistent activity pattern across motifs was present at each recording site.

\section{Unilateral HVc stimulation during singing leads to a rapid readjustment in activity of the contralateral HVc}

Briefly stimulating one $\mathrm{HVc}$ during a song motif had a rapid effect on both the song and the neural activity in the contralateral HVc. As reported previously (Vu et al., 1994), unilateral HVc stimulation caused rapid suspension of an ongoing motif followed by rapid resumption of singing with the start of a new motif (Fig. 


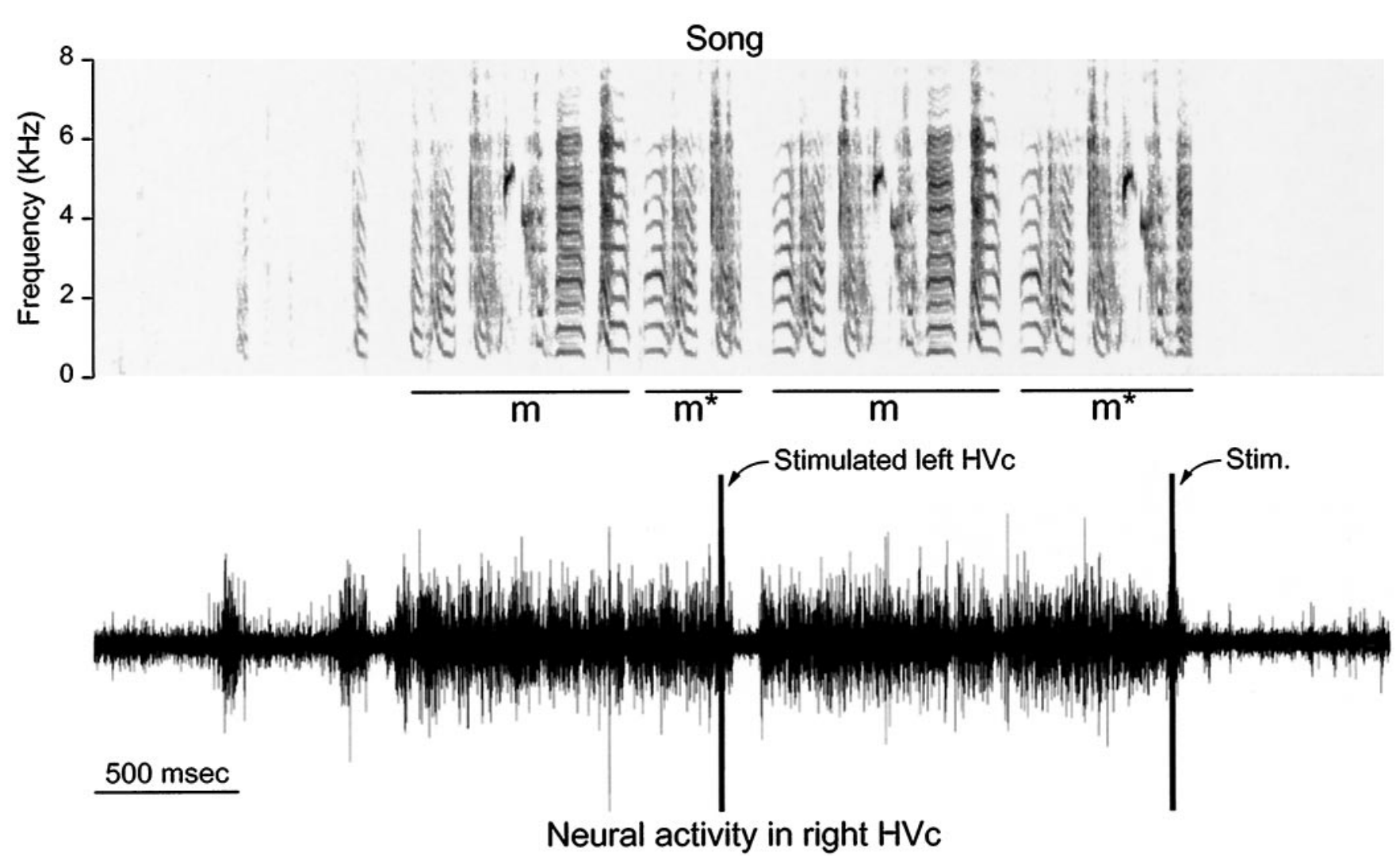

Figure 4. Perturbing neural activity in one $\mathrm{HVc}$ leads to a rapid change in the neural activity in the contralateral HVc. A brief electrical stimulus (5 pulses at $400 \mathrm{~Hz} ; 40 \mu \mathrm{A}$ ) applied to the left $\mathrm{HVc}$ during the second motif was sufficient to cause the bird to suspend the ongoing motif as well as to interrupt premotor activity in the contralateral $\mathrm{HVc}$; both neural activity and song resumed after $<100 \mathrm{msec}$. A second brief stimulus applied during the fourth motif caused the bird to suspend the ongoing motif and terminate song. Stim, Stimulation; $m$, song motif; $m *$, suspended song motif.

4, top). If the stimulus was applied late in the song (e.g., the second stimulus in Fig. 4) when the bird might have stopped singing at the end of the motif whether or not a stimulus was applied, the suspended motif sometimes was not followed by a new motif. The bottom trace in Figure 4 shows that at the same time that a stimulus in one $\mathrm{HVc}$ altered the song pattern, it also produced a rapid effect on the pattern of activity in the contralateral, unstimulated HVc.

Figure 5 illustrates more clearly the effect on neural activity that was observed. The "normal" mean activity associated with unstimulated motifs of one bird is shown in Figure $5 A$ (same bird as in Fig. 3). Figure 5, $B$ and $C$, show two examples of the effect that stimulating the left $\mathrm{HVc}$ had on neural activity in the right $\mathrm{HVc}$. The processed neural records were aligned to the onset of the first syllable of the motif in the sound records. In Figure $5 B$, the neural activity in the unstimulated right $\mathrm{HVc}$ was rapidly suppressed to baseline level (dotted line) after the end of the stimulus artifact in the record (compare neural record in Fig. $5 A$ for the expected level of activity over the same period if a stimulus had not been applied). The stimulus in this example had been delivered just before the vocalizing of syllable $d$ of the motif. The resulting syllable $d$ was distorted, the ongoing motif was suspended, and a new motif began sooner than it would have if the stimulus had not occurred. Note that premotor activity in the right $\mathrm{HVc}$ resumed just before the start of the new motif. Another example from the same recording site is shown in Figure $5 C$. Thus, a rapid readjustment in the pattern of activity in the recorded $\mathrm{HVc}$ occurred after brief stimulation of the contralateral HVc.
Every applied stimulus (to all seven birds) that led to either motif suspension with song resumption $(n=24)$ or song termination $(n=5)$ also always caused premotor activity in the contralateral $\mathrm{HVc}$ to be suppressed. Conversely, weak stimuli $(<40$ $\mu \mathrm{A} ; n=11$; delivered via the same electrodes in the $\mathrm{HVc}$ ) that did not alter the song pattern, presumably because not enough neurons in that $\mathrm{HVc}$ had been perturbed by the stimulus, also never affected the activity recorded in the contralateral HVc. These results suggest that the pattern of the song was changed only if the patterns of activity in both HVcs were altered.

The effects observed on neural activity in the recorded HVc were not caused by direct stimulation of its neurons (e.g., by the inadvertent return of stimulating currents via the recording electrode) because even stronger stimuli (60-70 $\mu \mathrm{A})$ delivered via stimulating electrodes that were deliberately placed $\sim 2 \mathrm{~mm}$ outside the borders of the HVc in two birds had no effect on the neural activity in the contralateral $\mathrm{HVc}$ (data not shown).

It was shown previously that unilateral stimulation of either the left or the right HVc altered the song pattern (Vu et al., 1994). Similarly, we found here that the pattern of premotor activity in either the right or the left $\mathrm{HVc}$ was rapidly altered by stimulation of the contralateral $\mathrm{HVc}$ (four right and three left HVcs were stimulated). This suggests that when premotor activity descending from the two hemispheres becomes temporally mismatched, the activity of both HVcs is subsequently "reset" by feedback mechanisms, and neither HVc can maintain its previous pattern of activity when a significant change in the pattern of activity in the contralateral HVc occurs. 

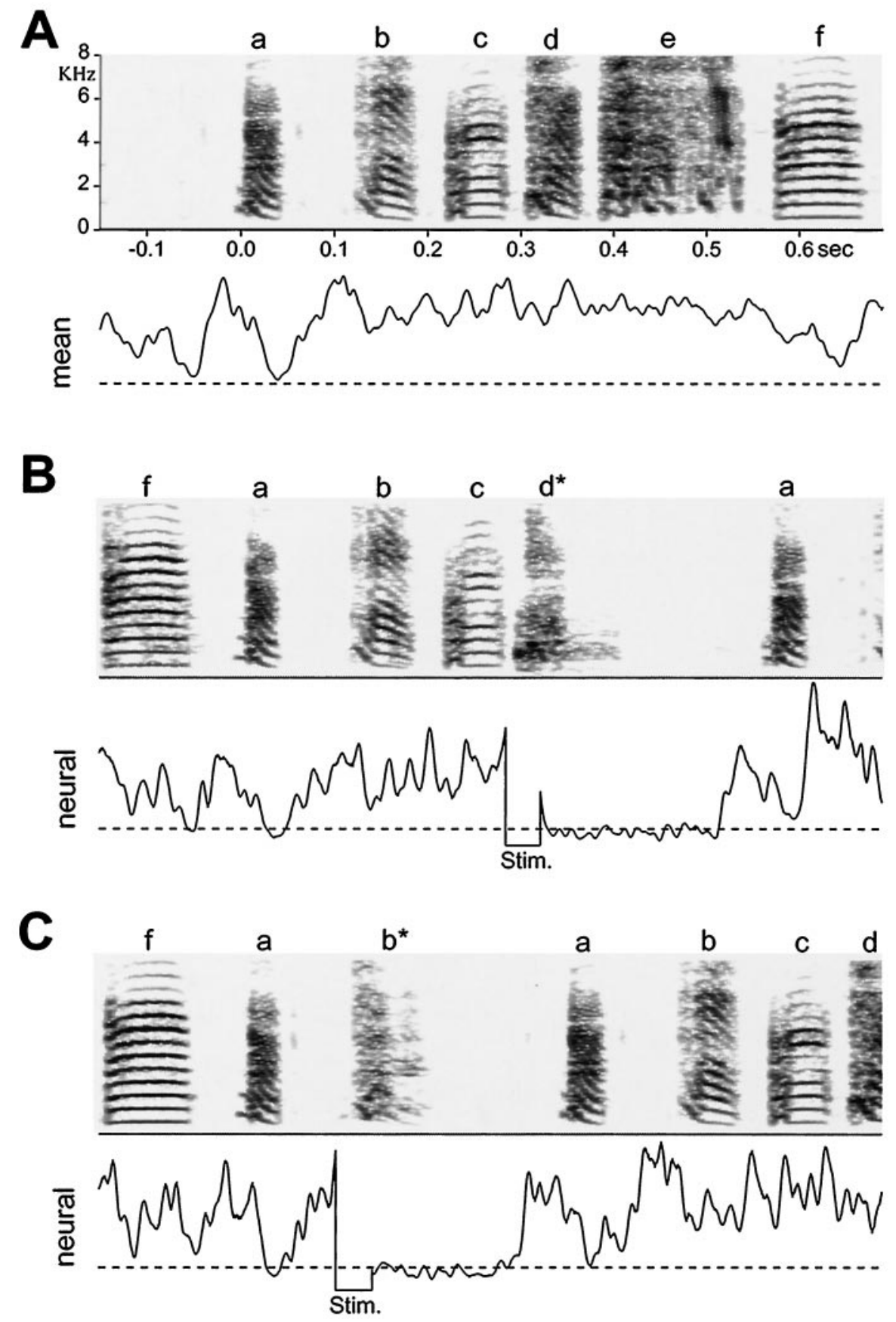

Figure 5. Effects of unilateral HVc stimulation on individual motifs and the corresponding activity in the contralateral HVc. $A-C$, Top, The spectrogram of individual motifs is shown. Bottom, The trace below each spectrogram represents either the mean pattern of processed neural activity observed across unstimulated motifs $(A)$ or simultaneously recorded neural activity in the right $\mathrm{HVc}(B, C)$. All neural records were aligned to the onset of the first syllable of the motif. Letters over each song syllable identify distinct syllable types; $b^{*}$ and $d^{*}$ indicate distorted syllables because of $\mathrm{HVc}$ stimulation. In $B$ and $C$, syllable $f$ of a prior motif can be seen at the start of the record. Stim, Stimulus artifact. The time scale in the spectrogram in $A$ applies to all other spectrograms and neural traces.

\section{Activity suppression after the stimulus was consistent during singing and specific for premotor activity}

To quantify the suppression of neural activity in the HVc after stimulation of the contralateral $\mathrm{HVc}$, we measured the level of activity above baseline over a period of $100 \mathrm{msec}$ after the end of the stimulus artifact, for each processed neural trace containing a stimulus. This level of activity was normalized by dividing it by the mean activity level at the same site over the same time period in normal motifs (Fig. 6A, middle trace divided by top trace). For comparison, the level of activity over the same portion of the motif in a randomly chosen unstimulated motif was also obtained and normalized (Fig. 6A, bottom trace divided by top trace). Such a paired control was necessary because each stimulus started at a different time relative to the beginning of the motif and because the normal pattern of activity associated with the motif was different for each bird. The normalized level of activity for 100 msec after a stimulus was significantly less than the level over the same motif period in a paired control motif (Fig. 6B; $0.142 \pm$ 0.032 in stimulated motifs; $0.961 \pm 0.022$ in unstimulated motifs; $\left.t_{28}=-21.171 ; p<0.001\right)$. Thus, for at least $100 \mathrm{msec}$ after a stimulus in one $\mathrm{HVc}$, the neural activity in the contralateral $\mathrm{HVc}$ was consistently suppressed to a level that was on average 0.14 as much as would be expected if a stimulus had not been applied.

Complete suppression of $\mathrm{HVc}$ activity to the baseline level took a relatively short time to develop. The mean latency from the start of the stimulus to full suppression of $\mathrm{HVc}$ neural activity was $36.1 \pm 2.9 \mathrm{msec}$. The time of full suppression was defined as the first time point after the stimulus artifact at which neural 

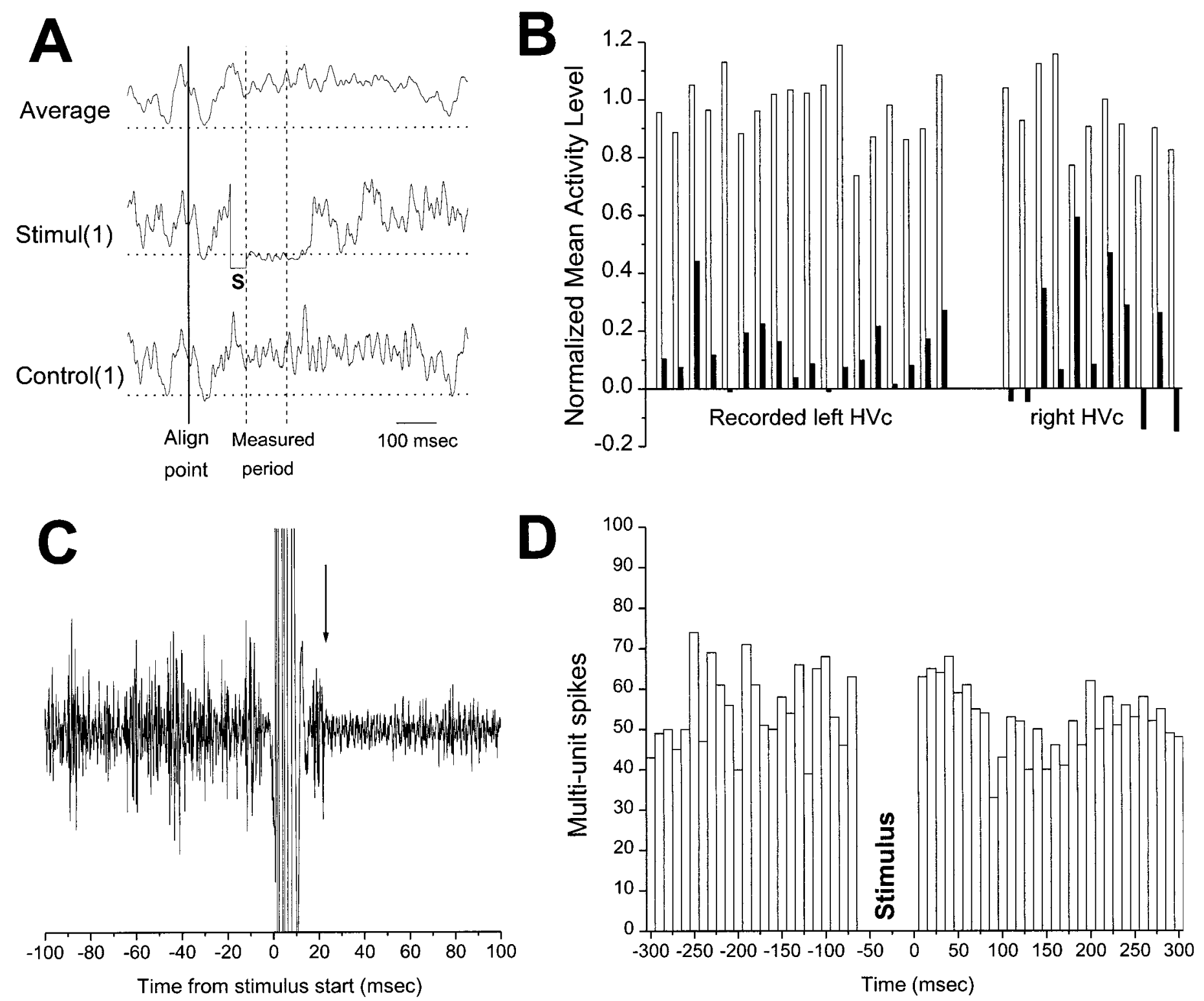

Figure 6. Suppression of HVc neural activity after stimulation of the contralateral HVc. A, Processed neural traces containing a stimulus [Stimul(1)] as well as randomly chosen and paired control traces [Control(1)] were aligned to the first syllable of the motif and compared with the mean pattern of neural activity observed across unstimulated motifs at the same recording site (Average) to determine the degree of suppression produced by the stimulus. The effect of stimulation was determined for a period of $100 \mathrm{msec}$ after the end of the stimulus artifact (Measured period). $S$, Stimulus artifact. B, Results from all traces containing a stimulus from nine recording sites in seven birds (filled bars; stimulated/average) and paired control traces (open bars; control/average), grouped according to whether the left or right $\mathrm{HVc}$ was recorded. The normalized activity level was negative in some cases because it is expressed relative to the baseline (nonsinging) activity level at the same recording site (see Materials and Methods). There was no significant difference between the degree of activity suppression of the left or right $\mathrm{HVc}$. $C$, Example of the latency to full activity suppression after stimulation in the contralateral HVc. Neural activity in this unprocessed trace is suppressed to the baseline level $\sim 24$ msec after stimulus onset (arrow). D, Peristimulus time histogram of multiunit activity in one $\mathrm{HVc}$ of a quiescent bird. Bin width was $10 \mathrm{msec}$; data from $25 \mathrm{stimuli}$ (aligned at the end of the stimulus). In this example, the duration of the stimulus train applied in the contralateral $\mathrm{HVc}$ was deliberately made longer (50 msec; $50 \mu \mathrm{A})$ than any stimulus applied during singing.

activity decreased to the baseline level (Fig. 6C, arrow). The shortest observed latency to full suppression was $\sim 24 \mathrm{msec}$ (Fig. $6 C)$. Although the latency to the start of suppression is not easily detectable with extracellular multiunit recording, it is probably shorter than $36 \mathrm{msec}$ on average because it must occur before the moment of full suppression.

One possible mechanism for activity suppression after the stimulus is the strong recruitment of inhibitory neurons (local and/or projecting to the $\mathrm{HVc}$ ), leading to inhibition of all pre- motor neurons in both HVcs. If this were the case, and if these inhibitory neurons could be activated by stimulating in one $\mathrm{HVc}$ whether the bird was singing or not, then some inhibition of spontaneous $\mathrm{HVc}$ activity might be detectable after stimulation in the contralateral $\mathrm{HVc}$ of quiescent birds. To test this, we applied, during nonsinging periods, the same type of stimulus that reliably induced activity suppression during singing and assessed the effect of this on the spontaneous activity in the contralateral HVc. 

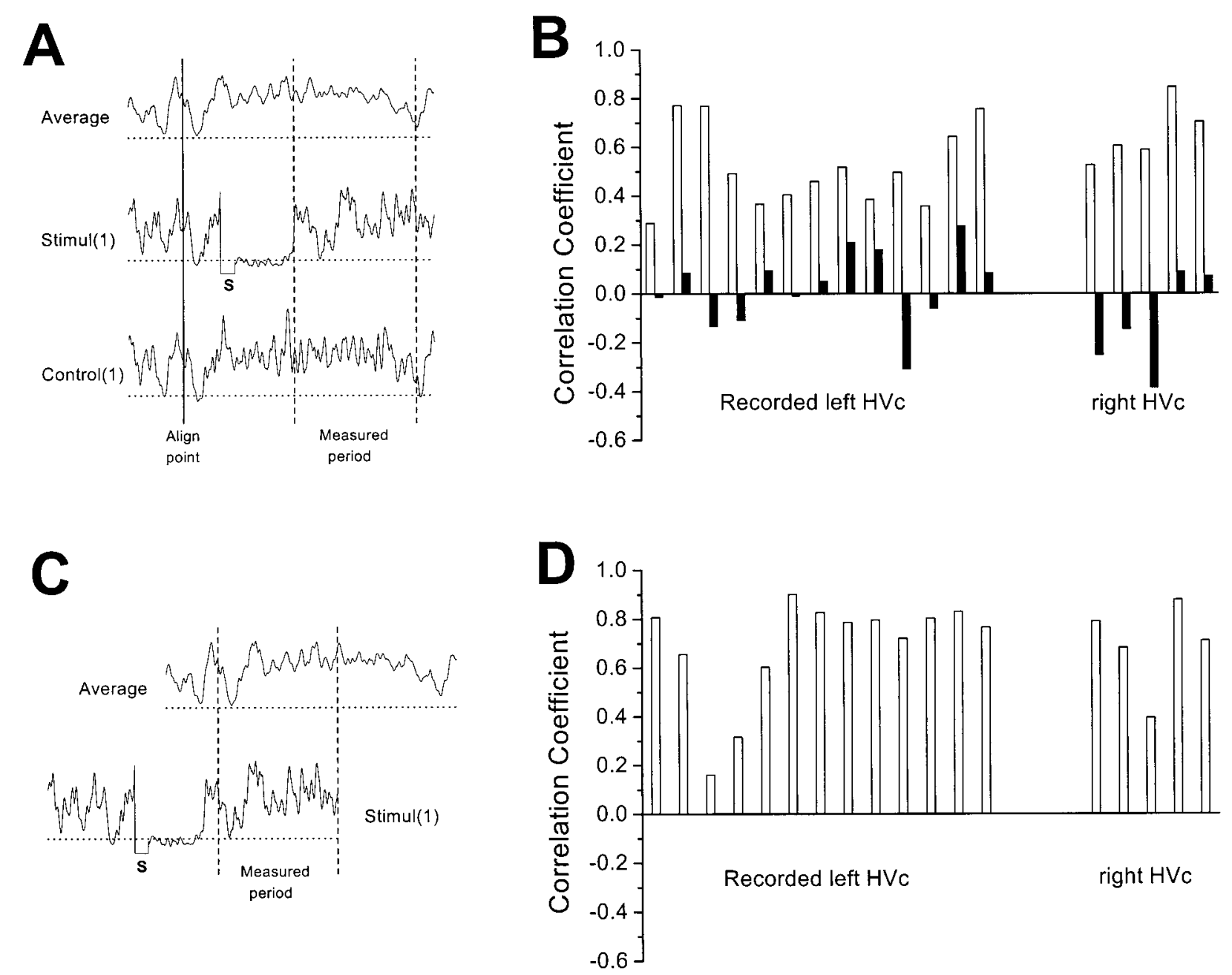

Figure 7. HVc activity resumes after contralateral stimulation with a different pattern from that predicted before the stimulus. $A$, Diagram of the period after each stimulus from which the pattern of neural activity was compared with the mean pattern observed across unstimulated motifs at the same recording site (Average trace). The comparison was made by calculating the linear correlation coefficient between pairs of traces. Same conventions as in Figure $6 A$. $B$, Results from all traces containing a stimulus in which activity resumed sufficiently rapidly to be compared with the end of the Average trace ( filled bars; stimulated vs average) and paired control traces (open bars; control vs average), grouped according to whether the left or right $\mathrm{HVc}$ was recorded. There was no significant difference between the degree of correlation of left or right $\mathrm{HVc}$ records. $C$, Diagram of the realignment of stimulated traces so that the onset of the first syllable in the resumed motif is aligned with the start of the motif in the Average record. $D$, Results from the same set of traces shown in $B$ after realignment of each stimulated trace with the Average trace.

Figure $6 D$ illustrates the results obtained at one recording site in the HVc. To increase the likelihood of detecting a decrease in the (baseline) level of activity in the $\mathrm{HVc}$ after the stimulus, we applied the stimulus 25 times (at $\sim 0.05 \mathrm{~Hz}$ ) while the bird was not singing and calculated the peristimulus time histogram of multiunit activity (see Materials and Methods). The spontaneous neural activity in the $\mathrm{HVc}$ during nonsinging periods was not altered significantly by stimulation of the contralateral $\mathrm{HVc}$, even when the duration of the stimulus train was increased to more than three times that applied during singing. For each of the 25 stimuli applied, we calculated the ratio of activity level for $100 \mathrm{msec}$ after the stimulus to the activity level for $100 \mathrm{msec}$ before the stimulus. The resulting mean ratio was $0.979 \pm 0.096$ and was not significantly different from a value of 1 . Similar results were obtained at a second recording site in the same bird and at a third recording site in a different bird. These results suggest that the activity suppression of $\mathrm{HVc}$ after the stimulus was specific for premotor activity because suppression was not detectable in the absence of premotor activity.

\section{The pattern of activity in the unstimulated HVc was reset and not just transiently suppressed}

The possibility existed that the only effect of stimulating one $\mathrm{HVc}$ on the premotor activity in the contralateral $\mathrm{HVc}$ was a transient suppression that otherwise did not affect the pattern of activity in the unstimulated HVc. If this were the case, then it would imply that although mechanisms exist to detect a mismatch in premotor output from the two hemispheres, no feedback mechanism is available to resynchronize the two sides.

To determine whether the pattern of activity in the unstimulated $\mathrm{HVc}$ actually changed after the transient suppression period, we compared the pattern of the resumed (after suppression) neural activity with the pattern of activity that would be expected if a stimulus had not been applied. Each processed neural trace containing a stimulus was aligned with the trace representing the normal mean activity of unstimulated motifs and with the neural trace associated with a randomly chosen unstimulated motif (Fig. $7 A$ ). The point of alignment was the onset of the first syllable of 
the motif in the corresponding sound records. The time of resumption of activity after the stimulus, relative to the alignment point, was determined as the time at which activity first increased to 1.5 times the baseline level after the suppression period (Fig. $7 A$, left vertical dashed line). Neural traces were compared from this time of resumption with a time point that was $30 \mathrm{msec}$ from the mean end of unstimulated motifs in the sound records (Fig. $7 A$, right vertical dashed line). The linear correlation coefficient $r$ was obtained between each "stimulated" trace and the corresponding mean trace, as well as between each paired control trace and the mean trace. Data from eleven cases were not used in this analysis either because the stimulus had been applied late in the song and resulted in song termination or because the stimulus had been applied near the end of the motif and the ensuing suppression period extended beyond the normal end of the motif.

Correlations between stimulated and normal mean traces were very low $(r,-0.015 \pm 0.043)$ and significantly different from correlations between paired control traces and the mean traces (Fig. $7 B ; r, 0.553 \pm 0.039 ; t_{17}=10.211 ; p<0.001$ ). Thus, the pattern of neural activity that resumed in the HVc shortly after stimulation of the contralateral $\mathrm{HVc}$ was different from what would be expected if a stimulus had not been applied, indicating that the premotor activity in the unstimulated $\mathrm{HVc}$ was truly reset rather than just transiently suppressed.

The resumed activity after the stimulus was always associated with the start of a new motif in the song (e.g., Fig. 5). Therefore, it seemed likely that the pattern of this resumed activity represented the normal activity pattern associated with the beginning of motifs. To confirm this, we compared again the same segment of resumed activity in each stimulated trace with the corresponding normal mean trace, but this time after realigning the traces so that the start of the new motif in the stimulated record was aligned with the start of the motif in the normal mean record (Fig. $7 C$ ). The resulting correlations were significantly higher than those obtained above with a different alignment point (Fig. 7D; $r$, $\left.0.69 \pm 0.048 ; t_{17}=-13.316 ; p<0.001\right)$. Thus, just as the temporal pattern of a song is rapidly reset to the beginning of a new motif by brief unilateral HVc stimulation (Vu et al., 1994), the pattern of premotor activity in the unstimulated contralateral $\mathrm{HVc}$ is also reset to that which corresponds to the beginning of motifs.

\section{DISCUSSION}

The main finding of this study is that unilaterally stimulating $\mathrm{HVc}$ in singing zebra finches leads to a rapid readjustment in the firing pattern of neurons in the contralateral HVc. This readjustment consists of a true resetting of the temporal pattern of activity in the contralateral $\mathrm{HVc}$ rather than merely a transient suppression of activity overlaid on an unaltered firing pattern. These results begin to describe the specific neural mechanisms that coordinate premotor activity in the two hemispheres during singing and raise the possibility that these mechanisms might play a role in the process of song learning.

\section{On-line monitoring and feedback control during singing}

The neural network mediating birdsong production is a bilateral network that includes discrete groups of neurons in the forebrain. During singing, premotor activity is observed in these forebrain song nuclei on both sides (McCasland, 1987; Schmidt, 1997), and this neural activity is temporally patterned rather than tonically active (McCasland and Konishi, 1981; Williams and Vicario,
1993; Yu and Margoliash, 1996) (the present study). Thus, premotor signals descending from the forebrain are thought to convey specific motor commands for the production of specific song elements. Consistent with this, briefly perturbing the activity in either $\mathrm{HVc}$ rapidly alters the subsequent song pattern $(\mathrm{Vu}$ et al., 1994), thus indicating that the spatiotemporal pattern of activity in the $\mathrm{HVc}$ contributes directly to the temporal pattern of the song.

Motor commands descending from the forebrain can potentially direct muscular activity on both sides of the body because sparse projections exist from RA to all contralateral midbrain and brainstem components of the song control network (Wild, 1994). If the song control nuclei on one side of the forebrain can control the respiratory and syringeal muscles on both sides of the body, why is it necessary to have these nuclei on both sides of the forebrain and to have both sides of the forebrain participate during singing? The answer may lie in the observation that some motor commands appear not to be conveyed contralaterally, because songbirds often control the left and right halves of their vocal apparatus, the syrinx, differently at the same time (Suthers, 1990; Allan and Suthers, 1994; Goller and Suthers, 1995). This is possible because the syringeal muscles on each side are innervated exclusively by the ipsilateral tracheosyringeal portion of the hypoglossal nucleus (Nottebohm et al., 1976; Vicario and Nottebohm, 1988).

The acoustic structure of individual adult song syllables is highly stereotyped from rendition to rendition. This implies that the patterns of muscular activity on the two sides of the syrinx during the production of each syllable must be well coordinated with each other as well as stereotyped. Direct evidence that the motor patterns on the two sides of the syrinx are precisely coordinated during singing has been obtained in gray catbirds, brown thrashers, and cowbirds (Suthers, 1990; Allan and Suthers, 1994; Goller and Suthers, 1995, 1996a,b). Given that temporally specific motor commands to syringeal motoneurons descend from song nuclei in the forebrain, then the requirement for precise bilateral coordination extends to the premotor activities on the two sides of the forebrain as well. However, this presents a special problem for songbirds because the song control nuclei in the forebrain are not directly interconnected across the midline (Fig. 1).

In theory, coordination of the premotor activities in the two hemispheres could be accomplished without requiring reciprocal connections between forebrain song nuclei across the midline. One possible mechanism is to initiate synchronously two independent but highly stereotyped motor programs, one in each hemisphere (Williams and Vicario, 1993). Thus, a single timing source could activate both hemispheres simultaneously to initiate a complete song or perhaps to initiate the production of a stereotyped sequence of syllables within the song, such as the motif in zebra finch songs (Sossinka and Böhner, 1980; Vu et al., 1994). The stereotypy of the motor programs on the two sides would ensure that vocal output is well coordinated bilaterally, despite the independence of the motor programs. This could be considered a strictly "feedforward" mechanism for bilateral coordination.

Interhemispheric coordination could also be accomplished by using both feedforward and feedback mechanisms. For example, it could be that, after synchronous initiation of the motor programs in the two hemispheres, the premotor outputs from the two hemispheres are continuously compared and mechanisms exist to resynchronize these when a sufficiently large temporal mismatch is detected. This on-line monitoring of descending motor com- 
mands would need to occur in one or more midbrain or brainstem nuclei that receive bilateral inputs from $\mathrm{RA}$, and the process of resynchronization would require feedback signals to both hemispheres from brain areas caudal to the forebrain.

The present experiments were designed to determine whether interhemispheric coordination of premotor activities in forebrain song nuclei is accomplished only with feedforward initiation or whether feedback mechanisms are also involved. Once initiated synchronously, if the two hemispheres act independently for the period of time between initiating commands, then the pattern of neural activity in one $\mathrm{HVc}$ (on either side) should not be altered by a brief perturbation of the neural activity in the contralateral $\mathrm{HVc}$ if this perturbation is applied between initiating commands. In contrast, if interhemispheric coordination is continuously maintained, then forcing the premotor activities in the two hemispheres out of synchrony at any time during a song (by electrically stimulating one $\mathrm{HVc}$ ) should lead to a short-latency adjustment of activity in the unstimulated HVc.

The latter results were observed in this study, thus providing the first physiological evidence that the two hemispheres do not act independently from each other at any time during singing and therefore that more complex mechanisms are involved in interhemispheric coordination than just synchronous initiation (Williams and Vicario, 1993).

\section{Organization and possible substrates of mechanisms for interhemispheric coordination}

The present results strongly suggest that the outputs of forebrain song control nuclei are continuously monitored and that an active mechanism exists for resynchronizing the premotor activities in the two hemispheres whenever a mismatch in their timing signals occurs. It remains to be determined directly whether the feedback mechanisms suggested here involve proprioceptive, somatosensory, auditory, or "internal" feedback (corollary discharge) or a combination of these, although a role for auditory feedback in the resetting mechanism is unlikely because unilateral HVc stimulation in deaf zebra finches also readily alters the temporal pattern of their songs (Vu et al., 1994).

The involvement of any type of sensory feedback in the resetting mechanism is not supported by a comparison between the latency from stimulation to readjustment of firing activity in the contralateral $\mathrm{HVc}$ and the expected time for motor commands from the $\mathrm{HVc}$ to reach the periphery and for sensory feedback to reach the forebrain. The shortest time observed from the start of the stimulus to the full suppression of HVc activity to the baseline level was $24 \mathrm{msec}$ (mean $=36.1 \mathrm{msec}$ ). This provides the upper limit of the latency to activity suppression because a small amount of time would be expected from the initiation of suppression to the full suppression to baseline in the HVc. In contrast, it would take at least $35 \mathrm{msec}$ from the start of $\mathrm{HVc}$ stimulation for motor output produced by that stimulation to reach the periphery (McCasland, 1987; Yu and Margoliash, 1996). This leaves insufficient time for any type of sensory feedback from the two sides to be processed and compared and for the result of this comparison to begin suppressing $\mathrm{HVc}$ activity. In the case of auditory feedback, it takes at least $13 \mathrm{msec}$ for auditory stimuli to affect the neural activity in the HVc (Margoliash, 1983; Margoliash and Fortune, 1992). Thus, it would require at least $48 \mathrm{msec}$ for activity to be readjusted in the contralateral $\mathrm{HVc}$ if only auditory feedback were used for interhemispheric coordination. In summary, these latency considerations suggest that interhemispheric coordination does not depend on sensory feedback and therefore is accomplished by comparing corollary discharges of premotor activities from the two hemispheres.

It was found previously that unilaterally perturbing RA is much less likely to alter the temporal pattern of an ongoing song than unilaterally perturbing $\mathrm{HVc}$ (Vu et al., 1994). This result would seem to contradict our hypothesis that a comparison of the premotor signals descending from the two hemispheres occurs downstream from both hemispheres, because stimulating one RA during singing should also result in a mismatch in premotor outputs from the two hemispheres (see Fig. 1). However, the mismatch resulting from stimulation in RA may be more restricted than that resulting from stimulating $\mathrm{HVc}$, because of the known functional segregation of RA projection neurons (Vicario, 1991). Thus, stimulation at one site in RA might perturb only a small subset of descending premotor commands, whereas the same kind of stimulation applied in $\mathrm{HVc}$ might have a more global effect on the descending commands from the stimulated hemisphere.

Alternatively, or additionally, there might be a threshold to the amount of time that descending commands are mismatched below which the mismatch is undetected and above which readjustment occurs. Thus, we suggest that the 15-msec-long stimulus train that was applied in RA (Vu et al., 1994) failed to reset the song pattern because it perturbed the activity in RA for only $\sim 15$ msec, and perhaps this was not long enough to activate the feedback pathways for resynchronization. We propose that the reason that the perturbation of RA activity did not outlast the duration of the stimulus is that temporally appropriate premotor signals from the HVc drove RA neurons to resume firing appropriately as soon as the stimulus in RA ended. In contrast, the same duration (or shorter) stimulus in the HVc might have resulted in a longer-lasting perturbation of HVc activity, leading to a mismatch in descending commands from the two hemispheres that was sufficiently long to activate resynchronization. Consistent with this hypothesis, stimulating RA with stimulus trains longer than $15 \mathrm{msec}$ did alter the song temporal pattern more frequently (E. T. Vu, M. E. Mazurek, and Y. Kuo, unpublished observations).

If the above explanation is true, then it would imply that the comparison of premotor outputs from the two hemispheres occurs at the level of gross temporal patterning (syllable durations and spacing) rather than fine detail (syllable structure). This might be desirable because it would allow motor commands to the two sides of the syrinx to differ in fine detail but be sufficiently synchronized not to trigger feedback resynchronization. At the same time, the differences in detail of the commands from the two hemispheres would allow differential control of the two sides of the syrinx, as has been demonstrated in other songbird species (Suthers, 1990; Goller and Suthers, 1995).

Three different anatomical pathways could convey singingrelated corollary discharge information back to both hemispheres. (1) Vates et al. (1997) described a pathway from RA to the dorsomedialis posterior nucleus of the thalamus (DMP), from DMP bilaterally to the medial magnocellular nucleus of the anterior neostriatum (mMAN), and from mMAN to HVc. (2) A region in the ventrolateral part of the rostral medulla receives a projection from RA and projects bilaterally to the thalamic nucleus uvaeformis (Uva), which projects to HVc (Okuhata and Nottebohm, 1992; Reinke and Wild, 1998; Striedter and Vu, 1998). Reinke and Wild (1998) recently named this ventrolateral area in the rostral medulla the paraambigualis nucleus. (3) Striedter and Vu (1998) described a pathway from RA to the dorso- 
medial nucleus of the intercollicular region (DM), from DM bilaterally to Uva, and from Uva to HVc. Whether and how much each of these pathways contributes to interhemispheric coordination remain to be determined.

\section{Implications of nonauditory feedback mechanisms on song learning}

The present results indicate that in adult songbirds, singing involves the continuous monitoring of premotor outputs from the two hemispheres and feedback mechanisms for the purpose of interhemispheric coordination. It will be important to determine whether these nonauditory feedback mechanisms also operate in young songbirds that are learning to sing. The same mechanisms might play a vital role during song learning because they could provide the song control network with internal feedback of motor commands. Because these feedback signals would be independent of auditory feedback, they might provide a means to compare motor intent (via corollary discharge signals) with actual motor performance (via auditory feedback). Furthermore, because it would take a certain amount of time for corollary discharge signals to be conveyed back to the forebrain, it is possible that they would arrive in the $\mathrm{HVc}$ at about the same time as the auditory feedback resulting from those motor commands that generated the same corollary discharges. This would ensure a temporally accurate comparison between motor commands and their peripheral effects. In this respect, further research on the organization of the song control network may provide valuable insights into how motor learning networks are generally organized.

\section{REFERENCES}

Allan SE, Suthers RA (1994) Lateralization and motor stereotypy of song production in the brown-headed cowbird. J Neurobiol 25:1154-1166.

Goller F, Suthers RA (1995) Implications for lateralization of bird song from unilateral gating of bilateral motor patterns. Nature 373:63-66.

Goller F, Suthers RA (1996a) Role of syringeal muscles in gating airflow and sound production in singing brown thrashers. J Neurophysiol 75:867-876.

Goller F, Suthers RA (1996b) Role of syringeal muscles in controlling the phonology of bird song. J Neurophysiol 76:287-300.

Gurney ME (1981) Hormonal control of cell form and number in the zebra finch song system. J Neurosci 1:658-673.

Hartley RS (1990) Expiratory muscle activity during song production in the canary. Respir Physiol 81:177-188.
Lewicki MS (1996) Intracellular characterization of song-specific neurons in the zebra finch auditory forebrain. J Neurosci 16:5854-5863.

Margoliash D (1983) Acoustic parameters underlying the responses of song-specific neurons in the white-crowned sparrow. J Neurosci 3:1039-1057.

Margoliash D, Fortune ES (1992) Temporal and harmonic combinationsensitive neurons in the zebra finch's HVc. J Neurosci 12:4309-4326.

McCasland JS (1987) Neuronal control of bird song production. J Neurosci 7:23-39.

McCasland JS, Konishi M (1981) Interaction between auditory and motor activities in an avian song control nucleus. Proc Natl Acad Sci USA 78:7815-7819.

Nottebohm F, Stokes TM, Leonard CM (1976) Central control of song in the canary, Serinus canarius. J Comp Neurol 165:457-486.

Okuhata S, Nottebohm F (1992) Nucleus Uva might be part of a feedback circuit for song processing. Soc Neurosci Abstr 18:527.

Reinke H, Wild JM (1998) Identification and connections of inspiratory premotor neurons in songbirds and budgerigar. J Comp Neurol 391:147-163.

Schmidt MF (1997) Interhemispheric synchronization of vocal premotor activity in songbirds. Soc Neurosci Abstr 23:244.

Sossinka R, Böhner J (1980) Song types in the zebra finch Poephila guttata castanotis. Z Tierpsychol 53:123-132.

Striedter G, Vu ET (1998) Bilateral feedback projections to the forebrain in the premotor network for singing in zebra finches. J Neurobiol 34:27-40.

Suthers RA (1990) Contributions to birdsong from the left and right sides of the intact syrinx. Nature 347:473-477.

Vates GE, Vicario DS, Nottebohm F (1997) Reafferent thalamo"cortical" loops in the song system of oscine songbirds. J Comp Neurol 380:275-290.

Vicario DS (1991) Organization of the zebra finch song control system: II. Functional organization of outputs from nucleus robust archistriatalis. J Comp Neurol 309:486-494.

Vicario DS, Nottebohm F (1988) Organization of the zebra finch song control system: I. Representation of syringeal muscles in the hypoglossal nucleus. J Comp Neurol 271:346-354.

Vu ET, Mazurek ME, Kuo Y (1994) Identification of a forebrain motor programming network for the learned song of zebra finches. J Neurosci 14:6924-6934.

Vu ET, Schmidt MF, Mazurek ME (1996) Interhemispheric coordination of premotor neural activity during singing in adult zebra finches. Soc Neurosci Abstr 22:151.

Wild JM (1994) The auditory-vocal-respiratory axis in birds. Brain Behav Evol 44:192-209.

Williams H, Vicario DS (1993) Temporal patterning of song production: participation of nucleus uvaeformis of the thalamus. J Neurobiol 24:903-912.

Yu AC, Margoliash D (1996) Temporal hierarchical control of singing in birds. Science 273:1871-1875. 